\title{
Alcohol effects on the variability of performance in a videogame task
}

\author{
LOWELL T. CROW and KATHY R. HIRDLER \\ Western Washington University, Bellingham, Washington
}

Subjects were given wine $(0.4 \mathrm{~g} / \mathrm{kg}$ ethanol) or no treatment prior to performing a novel videogame task. Data included total game scores as well as an uncertainty index (Frick \& Miller, 1951) of discrete-movement sequences of the paddle controller. Alcohol was shown to reduce the uncertainty of movements without affecting overall scores. In addition, sex-linked differences, which appeared in the uncertainty-of-response sequences under control conditions, were not observed in the alcohol-receiving group. The results are discussed as they relate to behavioral-variability theory (Crow, 1977).

One of the present authors has suggested that response variability is directly related to the functional complexity of the nervous system (Crow, 1977) and to the degree of mild stress (Crow, 1985). In rats, frontal lobe lesions (Crow \& McWilliams, 1979) as well as alcohol injections (Crow, McWilliams, \& Ley, 1979) were shown to reduce the variability of operant responding. In the latter study, alcohol was shown to reduce the uncertainty of response sequences as measured by an uncertainty index (Frick \& Miller, 1951). Also in rats, it was shown that signalled shocks reduced the variability of operant responding in a similar fashion (Crow, Bendt, \& Tracy, 1982).

The intent of the present study was to extend these findings to human behavior by exploring the effects of alcohol on complex motor-response sequences in videogame performance and, in addition, to note sex-related differences which may appear.

\section{METHOD}

\section{Subjects}

Subjects were 35 students from Western Washington University. There were 18 females, 5 of whom received alcohol, and 17 males, 8 of whom received alcohol. They ranged in age from 18 to 26 .

\section{Apparatus}

An Atari 800XL PC was used with an Amdek Color-I monitor and an Atari paddle controller. A videogame, "Paratroop Attack" (Plotkin, 1982), was modified to yield uncertainty scores from discrete movements of the knob and discrete trigger-button movements.

\section{Alcohol Doses}

Alcohol was given in the form of table wine in the amount of $0.4 \mathrm{~g} / \mathrm{kg}$ over a 30-min ingestion period.

\section{Videogame}

The game consisted of aiming a gun (knob of the paddle controller) and shooting with the button of the paddle controller at helicopters or

This paper was originally presented at the meetings of the Pacific Cascade Chapter of the Society for Neuroscience, Seattle, May, 1985. Correspondence should be addressed to the first author, Department of Psychology, Western Washington University, Bellingharn, WA 98225. paratroopers. Ten points were given per helicopter hit, and five points per paratrooper. A running score was displayed as part of the graphics. The duration of the game was 240 string elements (button responses + knob responses), or until a loss occurred. The game was lost if either seven paratroopers landed, or one landed directly upon the gun position.

\section{Procedure}

Subjects were asked to complete Spielberger's STAI forms X-1 and $\mathrm{X}-2$ and were given instructions on the videogame task. Alcohol-receiving subjects also completed an informed-consent statement and an alcoholdrinking-history statement prior to the game task.

Immediately prior to the game tasks, alcohol-receiving subjects were given $30 \mathrm{~min}$ to ingest the two to three glasses of wine required to reach the dosage level. Measures were taken for each of five games, the entire task requiring from 30 to $45 \mathrm{~min}$ to complete. All alcohol-receiving subjects were run in the afternoon immediately before returning home, and were escorted if driving was involved.

\section{RESULTS}

Randomized ANOVAs showed a significant difference between the alcohol-receiving and control groups for uncertainty scores on the game played approximately $30 \mathrm{~min}$ after alcohol ingestion $[F(1,32)=20.34]$. There were no significant differences in game scores between the alcoholreceiving and control groups $[\mathrm{F}(1,32)=.0006]$ on the test game (see Figure 1).

Although there was no significant difference in mean game scores (summed over all games played) between males and females, there was a significantly lower mean uncertainty score for females $[F(1,21)=42.45]$. In contrast, for the alcohol-receiving group there were no significant differences between the sexes in either mean scores or mean uncertainty scores for the test games.
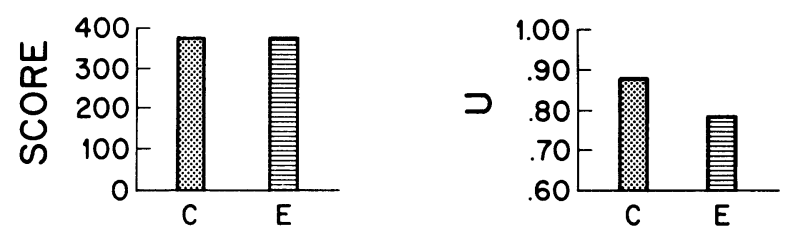

Figure 1. Mean scores (left) and mean uncertainty (right) of the control (C) and ethanol (E) groups. 
Correlation coefficients for the STAI-I and STAI-II and for the uncertainty scores (summed over all games) were $\mathrm{r}=-.12$ and $\mathrm{r}=.13$, respectively.

\section{DISCUSSION}

The findings of a reduced uncertainty score in the videogame performance as a result of alcohol ingestion is consistent with the earlier findings of a similar reduction in uncertainty scores in rats' operant behavior as a result of alcohol treatment (Crow et al., 1979). This consistency is in keeping with the expectations based on behavioral-variability theory (Crow, 1977).

The sex-linked differences in the control group's uncertainty scores were not expected, and are especially interesting in light of the fact that there were no sex-linked differences in game scores. In keeping with variability theory, an explanation of the lower uncertainty scores for females may be that videogame play was less familiar to women and therefore more stressful. Support for such an explanation was found in women's comments of incompetence at the game, which were much more common than those among men. That is, the women were more likely to report feelings of poor ability at the task, but scored as well as the men. The actual sex-linked differences lay in a greater stereotypy of responding on the part of women in manipulating the paddle controller to shoot down the player graphics.

Unexpected and inconsistent with variability theory was the lack of a substantial relation between stress-test scores and uncertainty scores. There were also no sex-linked differences in these stress scores, as has at times been reported.

The lack of a sex-linked difference in uncertainty scores in the alcohol condition may be due to the smaller number of subjects involved, but it is remarkable that a more homogeneous performance in terms of variability would be expected under alcohol conditions, from the perspective of variability theory. That is, individual variability (i.e., variability across individuals) as well as response variability (i.e., variability across responses) should be lessened by alcohol. In a time when the alcohol stress-reduction hypothesis is the subject of considerable controversy, it is interesting that stress reduction as a result of alcohol ingestion, if it does occur, may be better evidenced by variability data than by mean performance measures.

\section{REFERENCES}

Crow, L. T. (1977). Is variability a unifying behavioral concept? Psychological Record, 27, 783-790.

Crow, L. T. (1985). More on variability as a behavioral concept. Psychological Record, 35,293-300.

Crow, L. T., \& McWilliams, L. S. (1979). Relative stereotypy of water-ingestive behavior induced by frontal cortical lesions in the rat. Neuropsychologia, 17, 393-400.

Crow, L. T., McWilliams, L. S., \& Ley, M. F. (1979). Relative stereotypy of water-ingestive behavior induced by chronic alcohol injections in the rat. Bulletin of the Psychonomic Society, 14, 278-280.

Crow, L. T., Bendt, V. A. H., \& Tracy, D. M. (1982). Mutually antagonistic effects on behavioral variability of ethanol and an aversive CS+. Bulletin of the Psychonomic Society, 20, 263-265.

Frick, F. C., \& Miller, G. A. (1951). A statistical description of operant conditioning. American Journal of Psychology, 64, 20-36.

Plotkin, D. (1982). Textplot makes a game. In R. Lock (Ed.), Compute! Second Book of Atari (pp. 160-168). Greensboro: A Small System Services, Inc. 